\title{
A new classification model for online predicting users' future movements
}

\begin{abstract}
Nowadays many internet users prefer to navigate their interest web pages in special web site rather than navigating all web pages in the web site. For this reason some techniques have been developed for predicting userôs future requests. Data manning algorithms can be applied to many prediction problems. We can exploit Web Usage Mining for Knowledge extracting based on user behavior during the web navigation. The WUM applies data mining techniques for extracting knowledge from user log files in the particular web server. The WUM can model user behavior and, therefore, to forecast their future movements by mining user navigation patterns. To provide online prediction efficiently, we advance architecture for online predicting in web usage mining system by proposing novel model based on Longest Common Subsequence algorithm for classifying user navigation patterns. The prediction of usersôfuture movements by this manner can improve accuracy of recommendations.
\end{abstract}

